\title{
Restricted Expression of N- and R-Cadherin on Neurites of the Developing Chicken CNS
}

\author{
Christoph Redies, ${ }^{a}$ Hiroyuki Inuzuka, ${ }^{b}$ and Masatoshi Takeichi \\ Department of Biophysics, Faculty of Science, Kyoto University, Kitashirakawa, Sakyo-ku, Kyoto 606, Japan
}

\begin{abstract}
The expression of two cadherins, $\mathrm{N}$ - and R-cadherin, was mapped in the CNS of chicken embryos of 6-11 d incubation, focusing on the sensory and motor fiber systems. In the spinal cord, the laterally located fibers of the dorsal funiculus express $\mathrm{N}$-cadherin while the medially located fibers do not. These two fiber systems have a different course within the CNS but associate to form the spinal dorsal roots. In the hindbrain, $\mathrm{N}$-cadherin is expressed by the descending trigeminal (general somatic sensory) tract, which is contiguous with the N-cadherin-positive zone of the dorsal funiculus of the spinal cord. R-cadherin is not expressed by sensory fibers, but is expressed by the visceral motor system of the vagus and glossopharyngeal nerves, which are $\mathrm{N}$-cadherin negative. The motor neurites expressing R-cadherin have a different course within the brain than the sensory neurites expressing $\mathrm{N}$-cadherin, although they form the common sensory/motor roots of the vagus nerve at the surface of the brain.
\end{abstract}

The possibility that $\mathrm{N}$-cadherin provides a guidance cue for sensory axon migration within the CNS by a homophilic adhesion mechanism was investigated in vitro. Explants from sensory spinal ganglia expressing $\mathrm{N}$-cadherin were placed on $\mathrm{N}$-cadherin-transfected neuroblastoma cells, and axon outgrowth was visualized. Results showed that the sensory axons defasciculate and closely follow the cell-cell boundaries between transfected cells where high levels of $\mathrm{N}$-cadherin are expressed.

These results show that the two cadherins, like members of the immunoglobulin superfamily of molecules, are expressed in a topographically restricted fashion during chick brain development. They furthermore suggest that $\mathrm{N}$-cadherin expression by neurites may play a role in guiding these neurites along CNS paths that express the same molecule.

During the development of the CNS, axons grow along defined paths to their targets, sometimes over long distances. The molecular mechanisms guiding axonal growth have been under

\footnotetext{
Received Jan. 13, 1992; revised Mar. 30, 1992; accepted Apr. 2, 1992.

We thank Dr. H. Fujisawa for his generous gift of antibodies and Dr. H. Nakamura for his advice on the DiI experiments. This work was supported by research grants from the Ministry of Education, Science and Culture of Japan. C.R. was a rccipicnt of a fellowship from the Japan Society for the Promotion of Sciences.

Correspondence should be addressed to Dr. Masatoshi Takeichi, Department of Biophysics, Faculty of Science, Kyoto University, Kitashirakawa, Sakyo-ku, Kyoto 606, Japan.

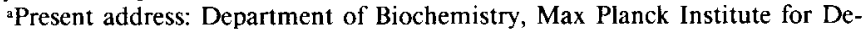
velopmental Biology, Spemannstrasse 35/II, W-7400 Tübingen, Germany.

bresent address: Biology Research Laboratories, Research and Developmental Division, Takeda Chemical Industries, Yodogawa-ku, Osaka 532, Japan.

Copyright (C) 1992 Society for Neuroscience $0270-6474 / 92 / 123525-10 \$ 05.00 / 0$
}

intense study in recent years. A number of developmentally regulated molecules that show a restricted pattern of expression in the developing CNS have been described. These molecules are thought to play important roles in growth cone elongation, axonal guidance, and fasciculation (for review, see Rutishauser and Jessell, 1988). Most of the molecules of this kind identified to date belong to the immunoglobulin superfamily of molecules. Examples are L1 (Rathjen and Schachner, 1984), fasciclin II (Grenningloh et al., 1991), fasciclin III (Patel et al., 1987), SC1 or DM-GRASP (Burns et al., 1991; Tanaka et al., 1991), TAG-1 (Dodd et al., 1988), Bravo (de la Rosa et al., 1990), and others. In this study, we show that members of another superfamily of molecules, the cadherins (Takeichi, 1988, 1991), are also expressed in the CNS in a temporally and topographically restricted pattern and may provide guidance cues for axon migration.

Cadherins are a family of molecules mediating $\mathrm{Ca}^{2+}-\mathrm{depen}-$ dent cell-cell adhesion in various tissues (Takeichi, 1988, 1991). A cadherin abundantly present during early neurogenesis, $\mathrm{N}$-cadherin (Hatta et al., 1987), was shown to play a role in neurulation (Hatta and Takeichi, 1986; Detrick et al., 1990; Fujimori et al., 1990), neuroepithelial morphogenesis (Matsunaga et al., 1988b; Takeichi et al., 1991), axon elongation (Matsunaga et al., 1988a; Tomaselli et al., 1988; Bixby and Zhang, 1990; Letourneau et al., 1990; Doherty et al., 1991), and axon fasciculation (Drazba and Lemmon, 1990).

Recently, evidence for the presence in brain of several other cadherins was found (Inuzuka et al., 1991a,b; Napolitano et al., 1991; Ranscht and Dours-Zimmermann, 1991; Suzuki et al., 1991). The role of these different cadherins in CNS development is unclear. In this work, we studied the expression of $\mathrm{N}$-cadherin, comparing it with that of another cadherin, R-cadherin (Inuzuka et al., 1991 a,b). We show that, during chicken CNS development [6-11 d of incubation (E6-E11)], these two molecules are differentially expressed in different fiber systems. The expression of the cadherins in specific fiber tracts suggests that cadherins may provide guidance cues for axon elongation. It is thus conceivable that axons expressing a specific cadherin may be guided to elongate along tracts expressing the same cadherin. This hypothesis was investigated in vitro by studying the pattern of axon outgrowth from $\mathrm{N}$-cadherin-positive sensory neurons on neuroblastoma cells expressing the same molecule.

\section{Materials and Methods}

Animals. Fertilized eggs from White Leghorn chickens were obtained from a local farm and incubated in a forced-draft incubator at $37^{\circ} \mathrm{C}$. Staging of the embryos was according to Hamburger and Hamilton (1951).

Generation of monoclonal antibodies against $R$-cadherin. R-cadherin fusion proteins were prepared as previously described (Inuzuka et al., 
199 la). Balb/c mice were immunized by intraperitoneal injection of the proteins ( $200 \mu \mathrm{g} /$ animal) emulsified with Freund's complete adjuvant. After boosting with the same amount of antigens emulsified with Freund's incomplete adjuvant, splenocytes of the mice were collected and fused with P3U1 myeloma cells. Culture supernatants of the hybridomas obtained were screened for reactivity to R-cadherin by immunoblotting. Two monoclonal antibodies, termed RCD- 1 and RCD-2, were isolated, and RCD-2 was used throughout the present experiments.

Immunocytochemicals. N-cadherin rat monoclonal antibody NCD-2 (Hatta and Takeichi, 1986) and R-cadherin mouse monoclonal antibody RCD-2 (see above) were partially purified by $50 \%$-saturated ammonium sulfate precipitation of hybridoma supernatant. Anti-chicken neurofilament mouse monoclonal antibody (Hatta et al., 1987) was a generous gift of Dr. H. Fujisawa. Appropriate secondary antibodies labeled with FITC, biotin, or Texas Red were purchased from Amersham. Streptavidin labeled with FITC or Texas Red (Amersham) was used to detect biotin-labeled secondary antibodies.

Immunostaining procedure. Immunostaining procedures were essentially as described elsewhere (Inuzuka et al., 199 la,b). Briefly, embryonic brains were fixed in freshly depolymerized $4 \%$ formaldehyde in Hank's buffered salt solution (HBSS), immersed in a graded scrics of sucrosc solutions ( $12 \%, 15 \%$, and $18 \%$ sucrose), embedded in Tissue Tek (Miles), and frozen in liquid nitrogen. Cryostat sections of 20-30 $\mu \mathrm{m}$ thickness were mounted and dried on slide glasses coated with chromium potassium sulfate and gelatin. Sections were postfixed in $4 \%$ formaldehyde/ HBSS and membranes permeabilized in $-20^{\circ} \mathrm{C}$ cold methanol. Unspecific binding was reduced by blocking the sections in a solution of Trisbuffered saline (TBS) containing $1 \mathrm{mM} \mathrm{Ca}{ }^{2+}, 5 \%$ skim milk, and $0.3 \%$ Triton X-100. For double-label immunostaining, sections were first incubated with one of the primary antibodies for $1-14 \mathrm{hr}$, followed by incubation with the appropriate fluorescently labeled secondary antibody (1-2 hr), and then postfixed for $10 \mathrm{sec}$ in an ethanol/acetic acid mixture $(95: 5 \% \mathrm{v} / \mathrm{v})$, and finally incubated with the second set of antibodies using the biotin-avidin detection system (Amersham). All antibodies were appropriately diluted in blocking solution. Ilybridoma supernatant was used after adding $0.3 \%$ Triton X-100. Fluorescence was visualized with a Zeiss epifluorescence microscope.

For whole-mount immunostaining, embryos of $6 \mathrm{~d}$ incubation were fixed in 4\% formaldehyde/HBSS and incubated overnight in TBS (pH 7.6) containing $1 \mathrm{mM} \mathrm{Ca}{ }^{2+}, 0.3 \%$ Triton $\mathrm{X}-100,1 \%$ bovine serum albumin, and $0.02 \%$ peroxide. The same solution without peroxide was then used for washes and antibody incubations, each lasting several hours to overnight. As the first antibody, NCD-2 was used. A biotinylated secondary antibody was detected by streptavidin-biotinylated horseradish peroxidase (Amersham). The diaminobenzidine reaction product was visualized under a Zeiss light microscope.

Dil experiment. For tract tracing in the spinal cord with Dil (Honig and Hume, 1986), chick embryos of $3 \mathrm{~d}$ incubation were transferred with the content of the egg into a Petri dish and incubated at $37^{\circ} \mathrm{C}$ in humid air following the procedure by Auerbach et al. (1974). The embryos were allowed to develop in vitro for $3 \mathrm{~d}$ until they had grown large enough for a small crystal of Dil (Molecular Probes) to be inserted at the cervical level into the dorsal portion of the spinal cord using fine pins. After 2 additional days of incubation, the embryos were fixed in $4 \%$ formaldehyde/HBSS and processed for immunohistochemistry as described above. Cross sections of the spinal cord were obtained through the area of implantation and at successively more caudal levels. Within 5 min after mounting cryostat sections on slide glasses, DiI was visualized and photographed under fluorescent illumination using a Zeiss microscope. Waiting for longer than about $5 \mathrm{~min}$ resulted in increased diffusion of DiI in the dried sections. As a control, consecutive sections were placed on slide glasses but immediately mounted in TBS without drying to prevent diffusion of DiI. Control results confirmed that there was little DiI diffusion in the dried sections before photography (data not shown). Dried sections were then processed for double-label immunohistochemistry using anti-neurofilament and NCD-2 antibodies as described above. Except for the implanted crystal of DiI, little or no Dil remained in the sections after immunostaining. The same sections that had been photographed earlier were analyzed again under epifluorescence illumination to obtain DiI, NCD-2, and neurofilament data from the same section.

cDNA transfection of neuroblastoma cells. Neuroblastoma cells of the Neuro 2a strain, which do not express any cadherin (Matsunaga et al., 1988a), were grown in a 1:1 mixture of Dulbecco's modified Eagle's medium and Ham's F12 medium supplemented with $10 \%$ fetal bovine serum and antibiotics (DH10). These cells were cotransfected with 1 $\mu \mathrm{g}$ of pMiwcN, a chicken N-cadherin expression vector (Fujimori et al., 1990), and $0.1 \mu \mathrm{g}$ of pSTneoB encoding the neomycin resistance gene (Katoh et al., 1987) in calcium phosphate precipitates. After selection with G418 as described (Hatta et al., 1988), two stable clones expressing $\mathrm{N}$-cadherin ( $2 \mathrm{a}-\mathrm{Ncad} \mathrm{l}$ and $2 \mathrm{a}-\mathrm{N}$ cad2) were obtained. Clones were tested for immunoreactivity with the monoclonal antibody NCD-2. As a control, two G418-resistant clones that did not express $\mathrm{N}$-cadherin were also selected (2a-01 and $2 \mathrm{a}-02)$.

Explant culture of sensory ganglia on $N$-cadherin-transfected cells. $\mathrm{N}$-cadherin-transfected and control neuroblastoma clones were plated on glass coverslips of $2.5 \mathrm{~cm}$ diameter placed in $3.5 \mathrm{~cm}$ culture dishes, at subconfluent density $\left(4-7 \times 10^{5}\right.$ cells/dish). Forskolin (WakoPure Chemicals) was added to the $\mathrm{DH} 10$ medium at a concentration of 0.03 $\mathrm{mM}$ to prevent clumping of the cadherin-transfected cells. The same medium was also used for the untransfected control cultures. One day after plating, the cells in each dish had grown to confluence and were used as a substrate for axon outgrowth.

Sensory ganglia from embryos of $6 \mathrm{~d}$ of incubation (E6; stage 28-29) were dissected under the microscope and cut into fragments of about $50-150 \mu \mathrm{m}$ in diameter. The fragments were placed onto the transfected or nontransfected neuroblastoma monolayers after the medium was replaced with fresh DH10 medium supplemented with $10 \mu \mathrm{g} / \mathrm{ml}$ of NGF, 2\% heat-inactivated chicken serum, and forskolin. Half of this medium was replaced with fresh medium every $12 \mathrm{hr}$. Forty to $48 \mathrm{hr}$ later, the cells were fixed in 4\% formaldehyde/HBSS for 10-20 min, washed in HBSS, and stained with the anti-chicken neurofilament antibody as described above.

In some experiments, the $\mathrm{IgG}$ fraction of a polyclonal rabbit antiserum against chicken $\mathrm{N}$-cadherin, or the $\mathrm{IgG}$ fraction of nonimmunized control serum, was added to the culture medium at a concentration of 20 $\mu \mathrm{g} / \mathrm{ml}$. The IgG fractions were purified according to standard procedures (Harlow and Lane, 1988). The sera were first precipitated with $50 \%$-saturated ammonium sulfate. The precipitate was then dissolved and passed over a Protein A-Sepharose column (Pharmacia). The eluate from this column was again precipitated with ammonium sulfate, and the precipitate was dissolved and dialyzed extensively against HBSS For each experiment, at least three independent trials were performed on different days.

\section{Results}

$N$-cadherin expression in the spinal cord

Figure $1 B$ shows the $N$-cadherin expression pattern in a cross section of the E8 spinal cord. N-cadherin expression is prominent in the dorsal funiculus of the white matter, the floor plate (Shiga and Oppenheim, 1991), the periventricular region, and the radial glia. Outside the spinal cord, $\mathrm{N}$-cadherin expression is seen in the dorsal root ganglia and the dorsal root (Hatta et al., 1987; Inuzuka ct al., 1991b).

A comparison with the neurofilament expression pattern of the same section (Fig. $1 A$ ) reveals that only the lateral but not the medial part of the dorsal funiculus is $\mathrm{N}$-cadherin positive. Figure 1, $C$ and $D$, shows at a higher magnification that some fascicles of the dorsal root express $\mathrm{N}$-cadherin while others do not. In general, the $\mathrm{N}$-cadherin-positive fascicles enter the spinal cord more ventrally and merge with the $\mathrm{N}$-cadherin-positive region of the dorsal funiculus. In contrast, the $\mathrm{N}$-cadherin-negative fascicles enter the spinal cord more dorsally and merge with the $\mathrm{N}$-cadherin-negative region either directly or after traversing the $\mathrm{N}$-cadherin-positive region of the dorsal funiculus (Fig. $1 E, F$ ). Similar results were obtained from E6 and E11 embryos.

\section{DiI labeling of spinal cord}

To examine whether the $\mathrm{N}$-cadherin-positive and -ncgative regions in the dorsal funiculus represent any particular structures of the spinal cord, we analyzed the relationship between the patterns of $\mathrm{N}$-cadherin expression and Dil tract tracing (Honig 

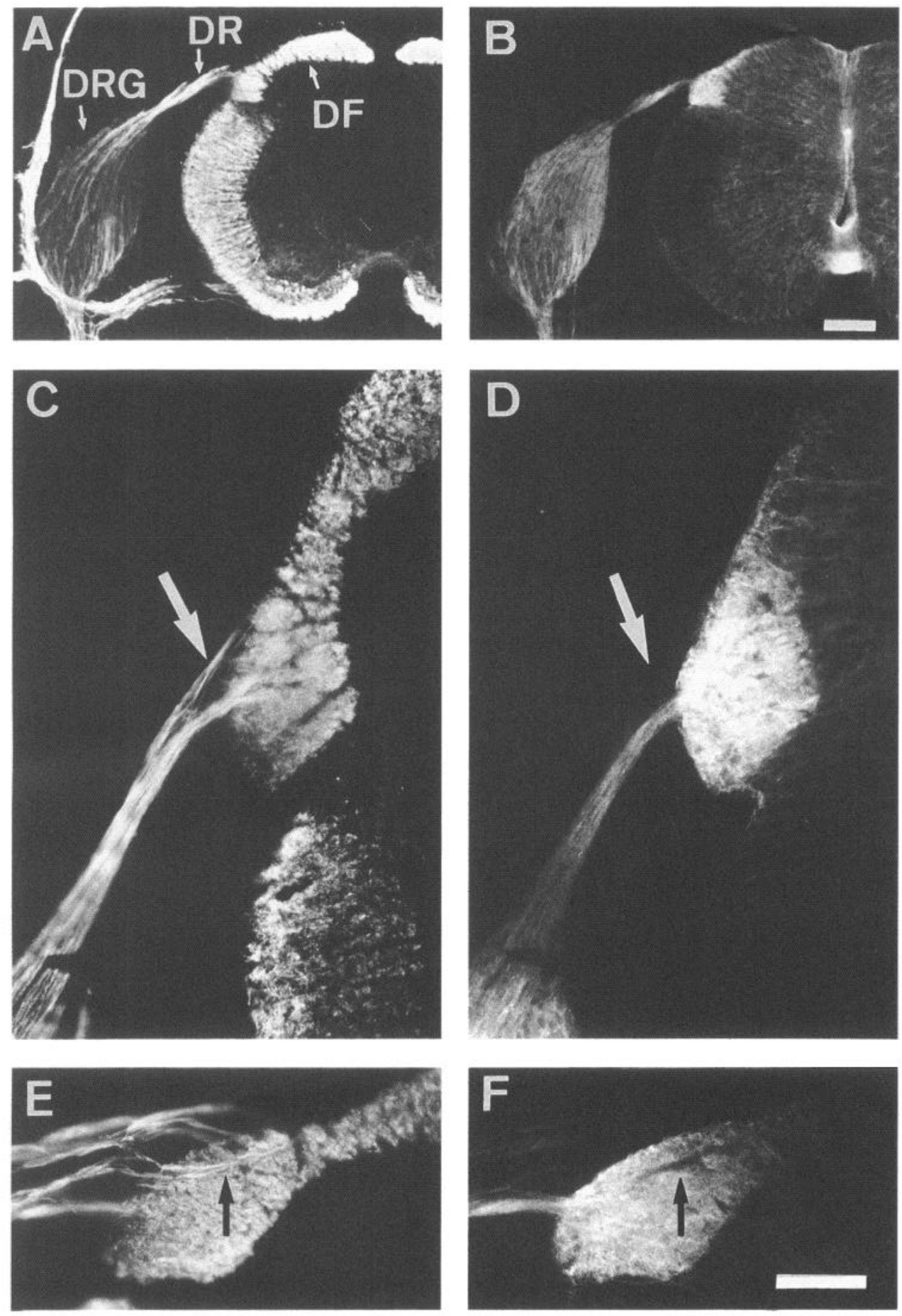

Figure 1. Double-staining for neurofilament $(A, C, E)$ and $\mathrm{N}$-cadherin $(B$, $D, F)$ in the thoracic spinal cord of E8 (stage 34) chicken. Note that only the lateral part but not the medial part of the dorsal funiculus expresses N-cadherin $(A, B)$. The arrows in $C$ and $D$ point to a dorsal root fascicle, which does not express $\mathrm{N}$-cadherin. The $a r$ rows in $E$ and $F$ point to an N-cadherinnegative dorsal root fascicle which runs through the lateral region of the dorsal funiculus which is $\mathrm{N}$-cadherin positive. $D R G$, dorsal root ganglion; $D R$, dorsal root; $D F$, dorsal funiculus. Scale bars: $B, 100 \mu \mathrm{m}$ for $A$ and $B ; F, 50 \mu \mathrm{m}$ for $C-F$. and Hume, 1986) of the sensory tract. DiI was implanted in the dorsal funiculus at a cervical level of E6 spinal cord, and the embryos were incubated for another $2 \mathrm{~d}$, sectioned, and stained for neurofilament and N-cadherin (Fig. 2). As shown in Fig. $2 A-C$, the entire dorsal funiculus is labeled with DiI at a level $0.5-1 \mathrm{~mm}$ caudal to the DiI implant. In contrast, DiI labeling is absent from the $\mathrm{N}$-cadherin-positive region at a level about $3 \mathrm{~mm}$ caudal to the implant (Fig. $2 D-F$ ). At this level, DiI is detected only in the $\mathrm{N}$-cadherin-negative region of the dorsal funiculus. In both cases, parts of the dorsal roots and the sensory ganglia also contain DiI. These results were confirmed in other embryos and indicate that the medial part of the dorsal funiculus contains longer distance projections than the lateral part.

\section{$N$-cadherin expression in the hindbrain}

Figure 3 shows a longitudinal section through the pons and medulla of an E8.5 embryo. Staining for N-cadherin (Fig. 3B) is seen only in a subset of the neurite fascicles that express neurofilament (Fig. 3A). The $\mathrm{N}$-cadherin-positive fibers extend in a continuous band from the entry of the trigeminal nerve at 

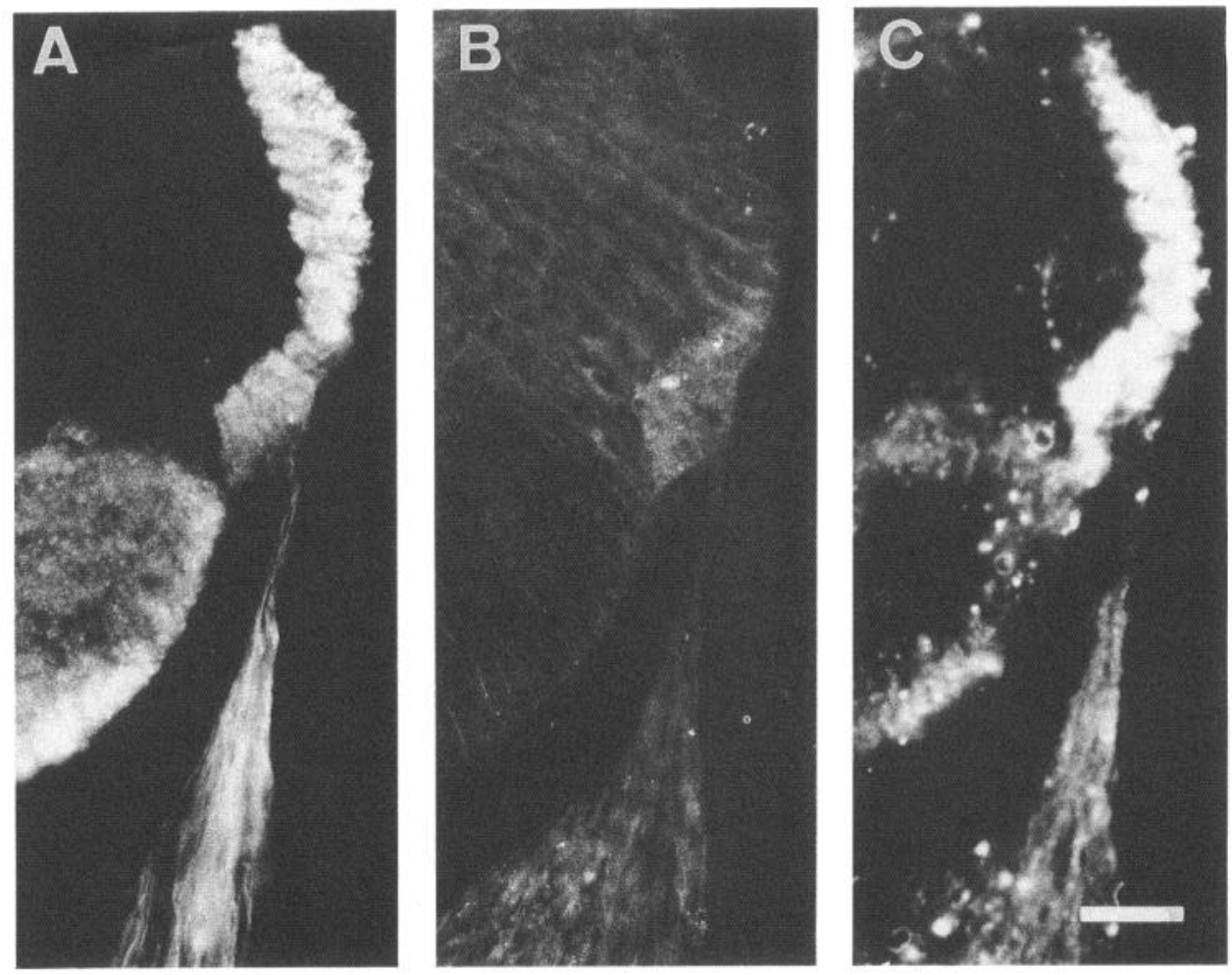

Figure 2. Triple-staining of E8 chicken spinal cord for neurofilament $(A, D)$, for N-cadherin $(B, E)$, and with DiI for tract tracing $(C, F)$. DiI crystals were implanted into the cervical spinal cord. The section shown in $A-C$ is $0.5-1 \mathrm{~mm}$ caudal to the DiI implant, and that shown in $D-F$ is about $3 \mathrm{~mm}$ caudal to the DiI implant. The asterisks in $D-F$ mark the lateral, $\mathrm{N}$-cadherin-positive region of the dorsal funiculus, which shows no Dil label at this level. At the level closer to the DiI implant $(A-C)$, the corresponding region contains DiI. Note that, at both levels, DiI label is present in the medial part of the dorsal funiculus. These sections are from an embryo incubated for $3 \mathrm{~d}$ in the egg and for another $5 \mathrm{~d}$ in a dish. Scale bars, 50 $\mu \mathrm{m}$.
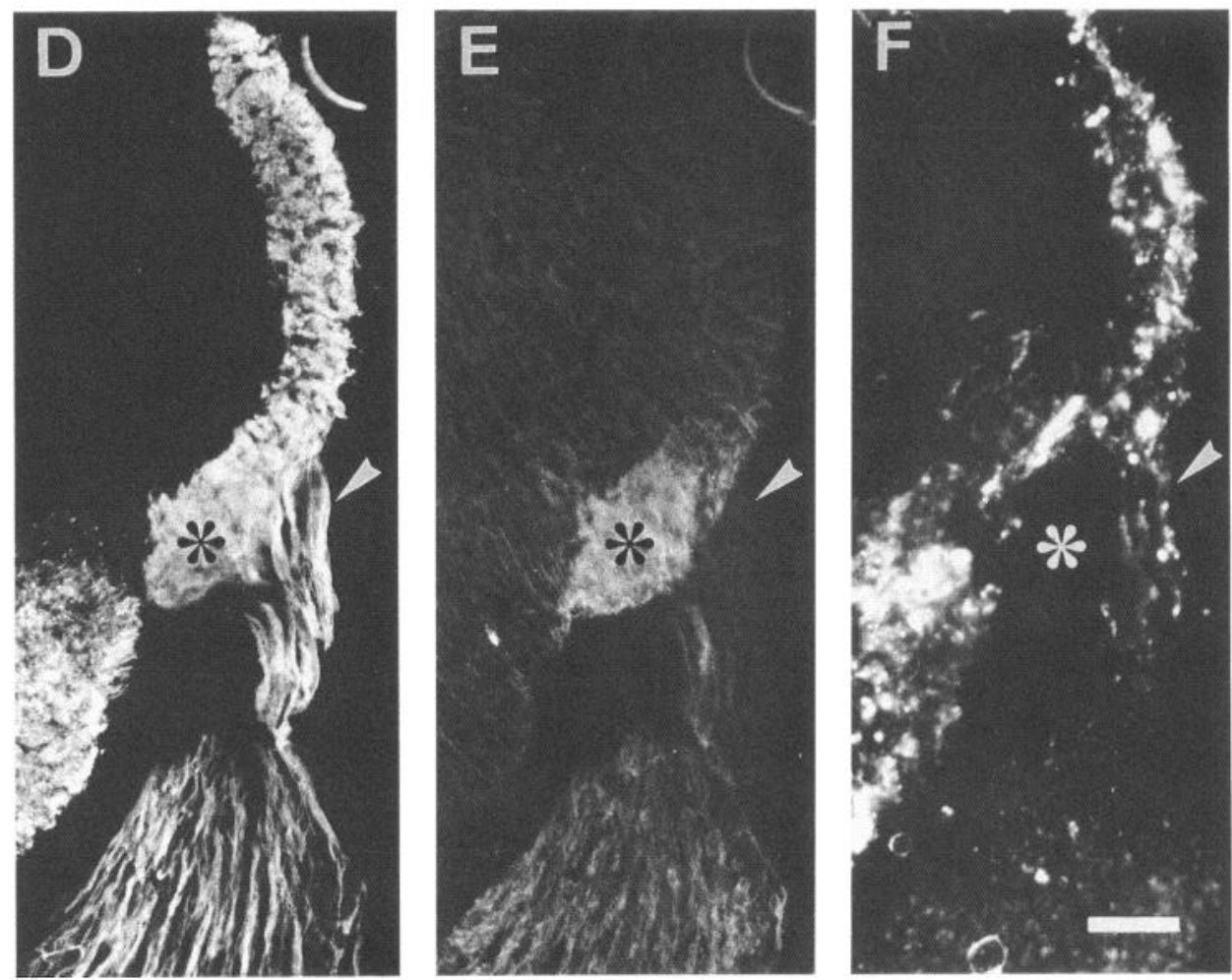

the rostral end of the pons to the caudal medulla oblongata. At a more caudal level, this $\mathrm{N}$-cadherin-positive fiber tract extends into the lateral part of the dorsal funiculus of the spinal cord (data not shown). In Figure 4A, the same fiber tract can be seen from a lateral viewpoint in a whole-mount preparation stained for $\mathrm{N}$-cadherin. Figures $3 B$ and $4 A$ show that the trigeminal, glossopharyngeal, and vagus nerves contain nerve fascicles expressing $\mathrm{N}$-cadherin, whereas in the fascial and cochleo-acoustic nerves, only glial elements are $\mathrm{N}$-cadherin positive.

In the trigeminal nerve, $\mathrm{N}$-cadherin-positive fascicles are found in the sensory portion but not in the motor portion (Fig. $4 B, C$ ). Furthermore, not all neurites in the sensory portion express 


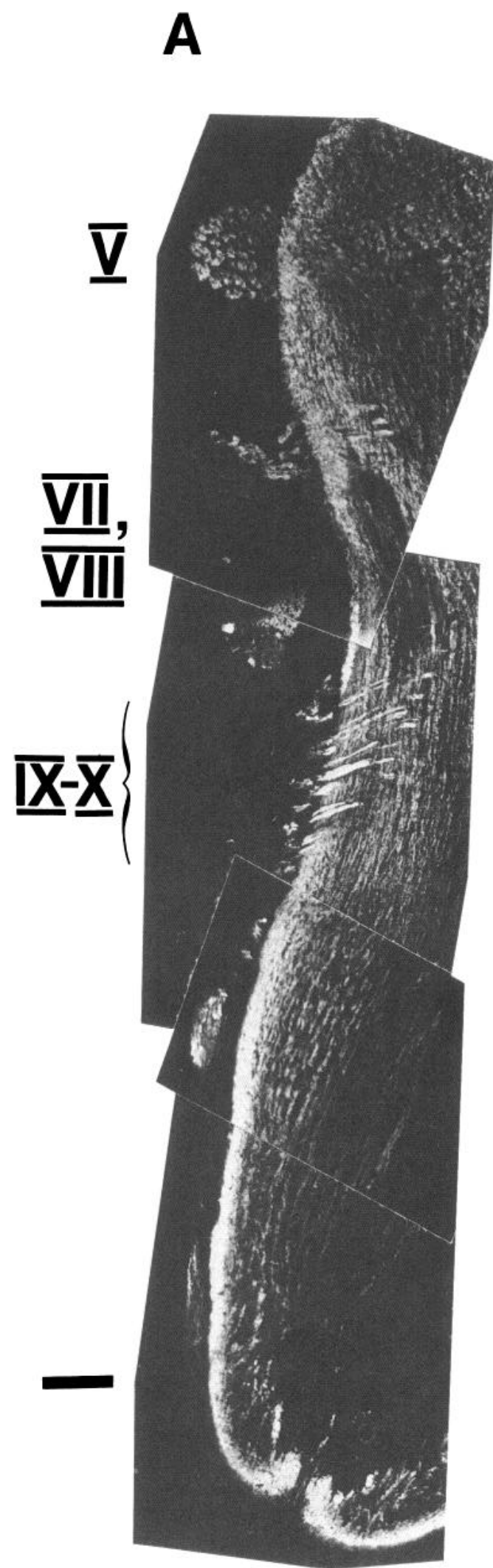

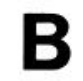

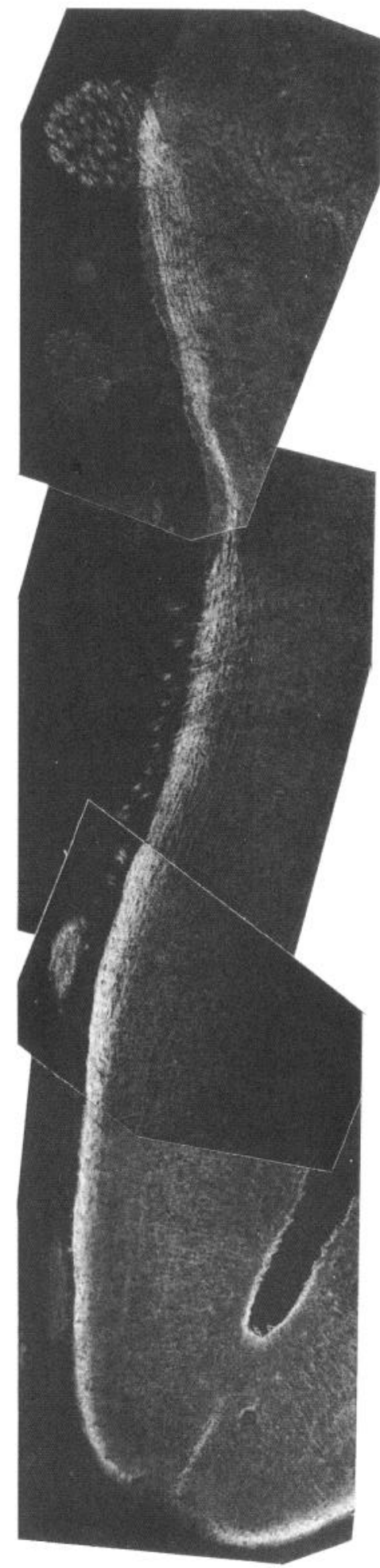

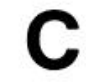
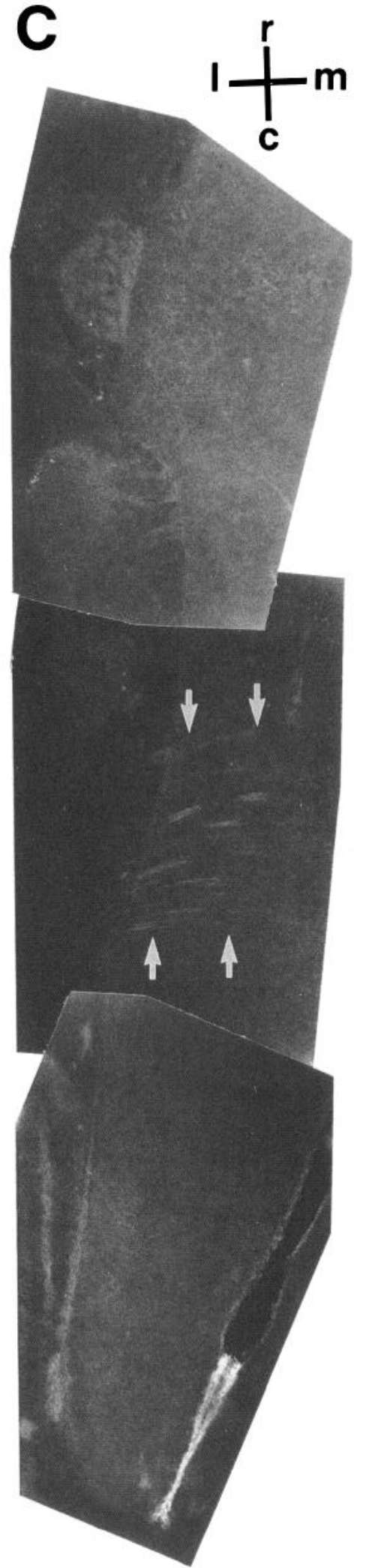

Figure 3. Double-staining of an E8.5 (stage 35) chicken hindbrain for neurofilament $(A)$ and N-cadherin $(B)$, and staining for R-cadherin $(C)$ in an adjacent section. These sections cut transversely through the pons and obliquely through the medulla oblongata (lower part of the sections). Arrows indicate R-cadherin-positive vagus motor fibers. $V$, root of trigeminal nerve; $V I I, V I I I$, roots of the facial and cochleo-acoustic nerves; $I X$ $X$, rootlets of the glossopharyngeal and vagus nerves; $r$, rostral; $m$, medial; $c$, caudal; $l$, lateral. Scale bar, $200 \mu \mathrm{m}$. 

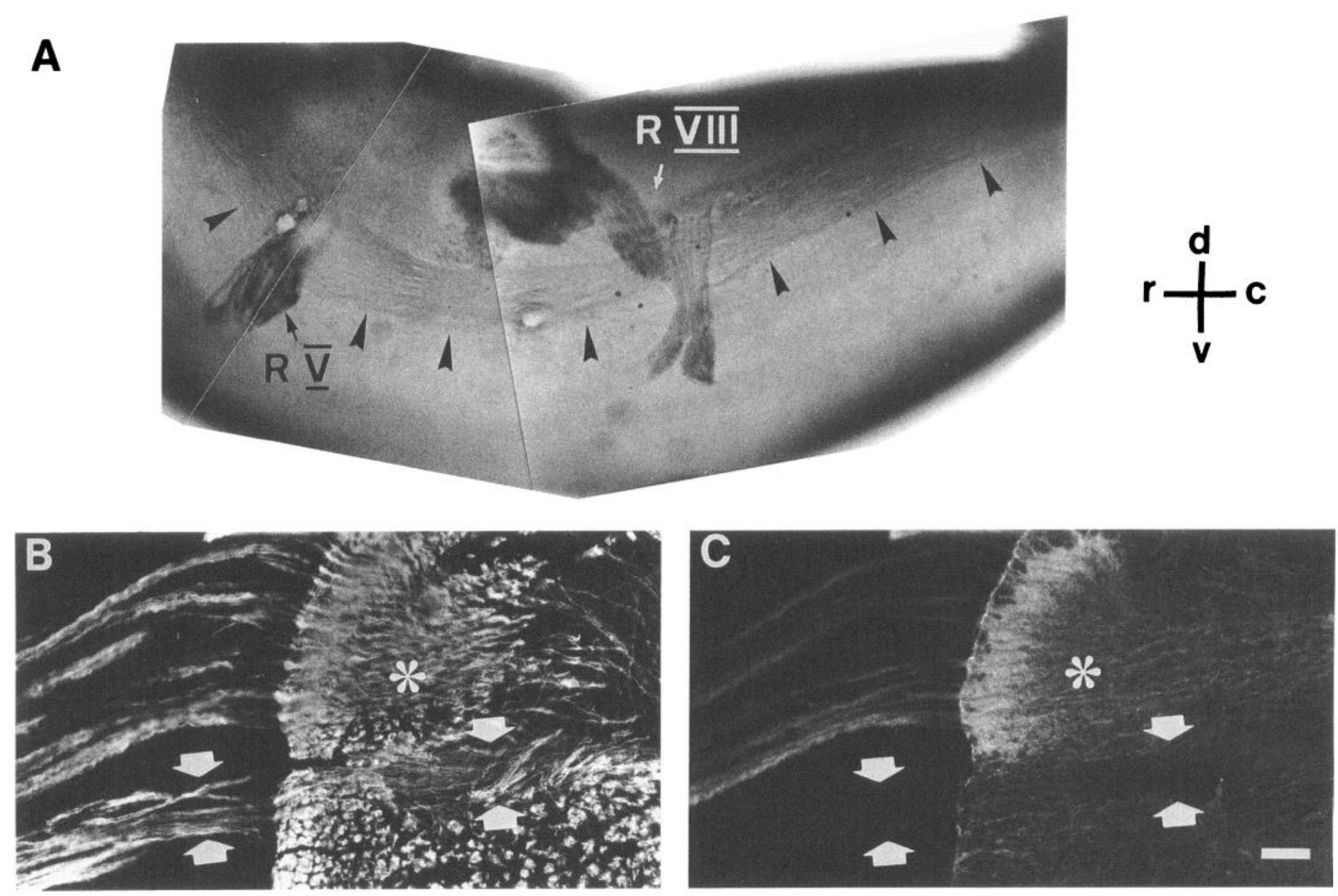

Figure 4. A, Lateral view of N-cadherin whole-mount staining of E6 (stage 29) chicken hindbrain. The arrowheads point to the ventral margin of the descending trigeminal tract. $R V$, root of the trigeminal nerve; $R V I I I$, root of the cochleo-acoustic nerve; $d$, dorsal; $c$, caudal; $v$, ventral; $r$, rostral. $B$ and $C$, Double-staining of an $\mathrm{E} 8$ (stage 34$)$ chicken for neurofilament $(B)$ and $\mathrm{N}$-cadherin $(C)$ in a cross section through the lateral pons at the level of the trigeminal nerve entry. The asterisk marks the region of the sensory trigeminal neurites that do not express $\mathrm{N}$-cadherin. Arrows point to the minor (motor) portion of the trigeminal nerve, which shows no N-cadherin immunoreactivity. Scale bars: $A, 200 \mathrm{~mm} ; B$ and $C, 50$ $\mu \mathrm{m}$.

$\mathrm{N}$-cadherin. At the entry zone of the trigeminal nerve, $\mathrm{N}$-cadherin staining is only observed in a comma-shaped zone directly below the surface of the pons. The neurofilament-positive fascicles that penetrate the pons more deeply do not express $\mathrm{N}$-cadherin. In the deeper regions, fine fibers expressing $\mathrm{N}$-cadherin but not neurofilaments are found. These fibers likely represent the processes of radial glia.

\section{$R$-cadherin expression}

$\mathrm{R}$-cadherin is expressed in various regions of the spinal cord, most strongly in the midline region. At the embryonic stages studied here, there is no R-cadherin expression in the sensory and motor fibers of the spinal and cranial nerves (Inuzuka et al., 1991b). However, we found one exception: R-cadherin is expressed in the motor nuclei of the vagus and glossopharyngeal nerves, which are located close to the midline (Fig. $5 A$ ), and in their processes that extend to the lateral surface of the brainstem (Fig. $3 C$ ). Here, they exit the brain by joining the $\mathrm{N}$-cadherinpositive (sensory) fibers that are entering the brain (Fig. 5B,C). The sensory fibers do not extend beyond the $\mathrm{N}$-cadherin-positive zone of the descending trigeminal tract (Fig. $3 B$ ), whereas the R-cadherin-positive (motor) fibers originating from midline structures reach the brain surface after traversing $\mathrm{N}$-cadherinnegative tissue (Fig. $3 \mathrm{C}$ ). A close inspection reveals that most of the fascicles in the vagus nerve rootlets are either R-cadherin positive or $\mathrm{N}$-cadherin positive but rarely both (Fig. $5 B-E$ ).

\section{Outgrowth of sensory axons on $\mathrm{N}$-cadherin substrate}

To test whether $\mathrm{N}$-cadherin in sensory axons is actively involved in their migration, E6 sensory ganglia were explanted on $\mathrm{N}$-cadherin-transfected and control cell lines (Fig. 6). On untransfected cells that do not express $\mathrm{N}$-cadherin (Fig. $6 \mathrm{~A}$ ), neurite outgrowth is strongly fasciculated. On N-cadherin-transfected cells (Fig. $6 B$ ), a strong defasciculation is observed that can be blocked by purified anti-N-cadherin IgG (Fig. $6 \mathrm{C}$ ). In those areas where defasciculation occurs, axons grow in a wavy, curved fashion (Fig. $6 B, D$ ). In its form and periodicity, this axonal growth pattern resembles the cell-cell boundary pattern of the $\mathrm{N}$-cadherin-transfected cells (Fig. $6 D, G$ ). Figure $6 E$ shows more clearly that the axons originating from sensory neurons follow the cell-cell boundaries. In the presence of anti-N-cadherin IgG, the axons grow much straighter (Fig. $6 F$ ). For comparison, the $\mathrm{N}$-cadherin expression pattern of the same cells is given in Figure $6 G$. 

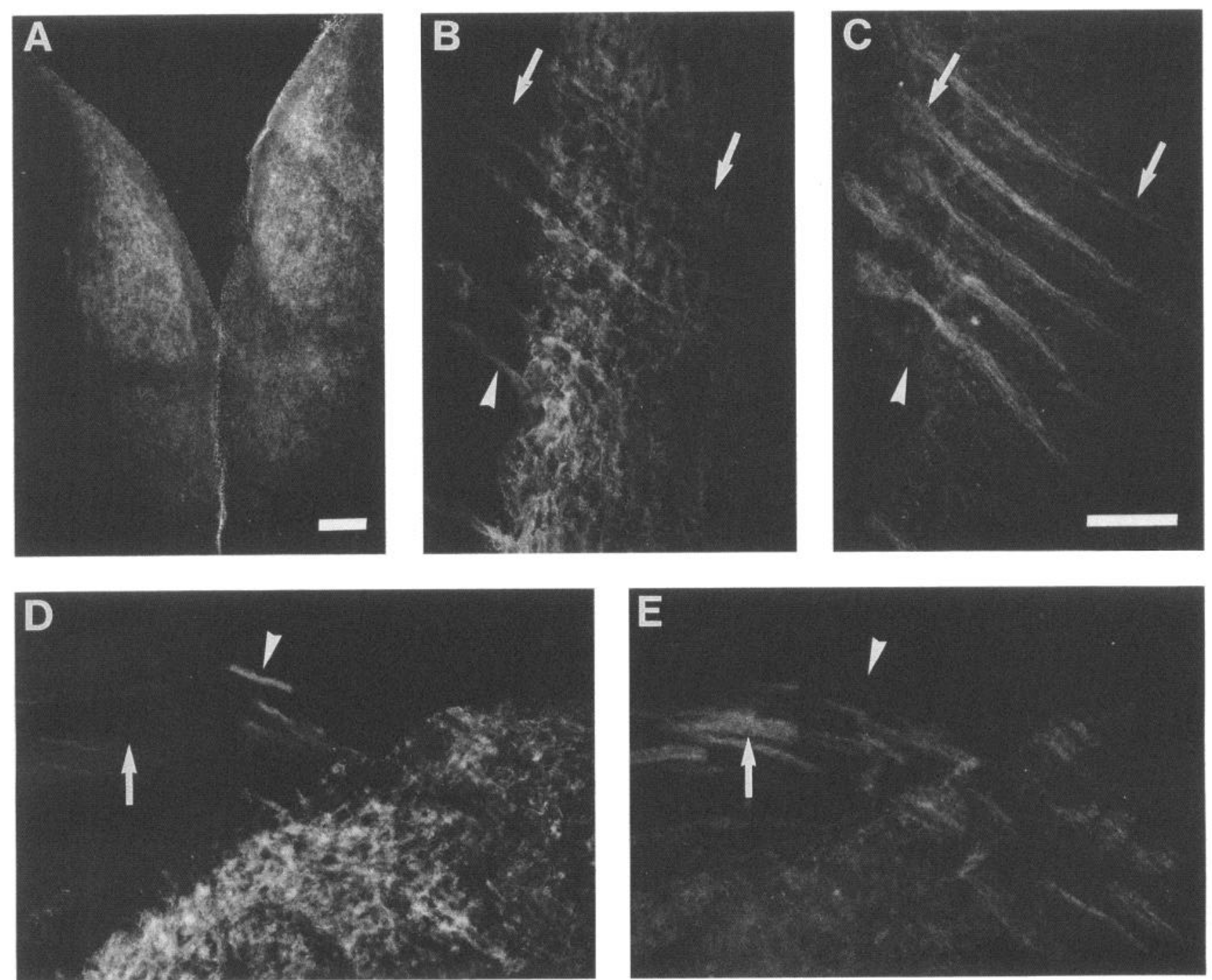

Figure 5. $\mathrm{N}$ - and R-cadherin expression in the vagus motor nuclei and nerve. A, R-cadherin staining of E11 (stage 37 ) medulla. This is a cross section through the midline with the ventricular surface facing up. Note the positive immunoreactivity in the motor nuclei of the vagus nerve. $B$ $E$. Double-staining for $\mathrm{N}$-cadherin $(B, D)$ and $\mathrm{R}$-cadherin $(C, E)$ of the vagus nerve at its entry point into the medulla. $B$ and $C$ are from a transverse section of the medulla from an E8.5 (stage 35) chick embryo. $D$ and $E$ are from a cross section from an E11 (stage 37 ) chick embryo. Arrows point to fascicles that express R-cadherin but not N-cadherin. Arrowheads point to fascicles that express N-cadherin but not R-cadherin. Scale bars: $A$, $50 \mu \mathrm{m} ; B-E, 100 \mu \mathrm{m}$.

\section{Discussion}

$N$-cadherin and $R$-cadherin expression are topographically restricted

In this study, we show that, in the CNS of E6-E11 chicken, $\mathrm{N}$-cadherin (Hatta et al., 1988) is expressed in a restricted fashion in a specific fiber tract, the general somatic sensory system (Figs. 1, 3-5). At the same time, another cadherin, R-cadherin (Inuzuka et al., 1991a,b), is expressed by a different system, the visceral motor component of the vagus and glossopharyngeal nerves (Figs. 3-5). These patterns of expression are observed at a time in development during which axons are actively growing. Little or very low levels of $\mathrm{N}$ - and R-cadherin are present in the adult brain (Inuzuka et al., 1991a; C. Redies and M. Takeichi, unpublished observations). It is conceivable that the other cadherins recently found in brain tissue (Napolitano et al., 1991; Ranscht and Dours-Zimmermann, 1991; Suzuki et al., 1991) are similarly distributed in a restricted, developmentally regulated way.

\section{$N$-cadherin is differentially expressed in two types of dorsal root fascicles}

$\mathrm{N}$-cadherin is expressed only in a subset of fascicles of the spinal dorsal root. In the dorsal funiculus into which most of the dorsal root fibers extend, only the lateral part is $\mathrm{N}$-cadherin positive. Figure 1 demonstrates that these two fiber systems are appropriately connected so that dorsal root fascicles that are positive for $\mathrm{N}$-cadherin join the lateral part of the dorsal funiculus while the negative neurites join the medial part.

The results from the DiI experiments suggest that these two portions of the dorsal funiculus are not equivalent but contain at least two different fiber systems. Whereas the medial part contains fibers extending rostrally over relatively large distances, the lateral, N-cadherin-positive region does not (Fig. 2). This 

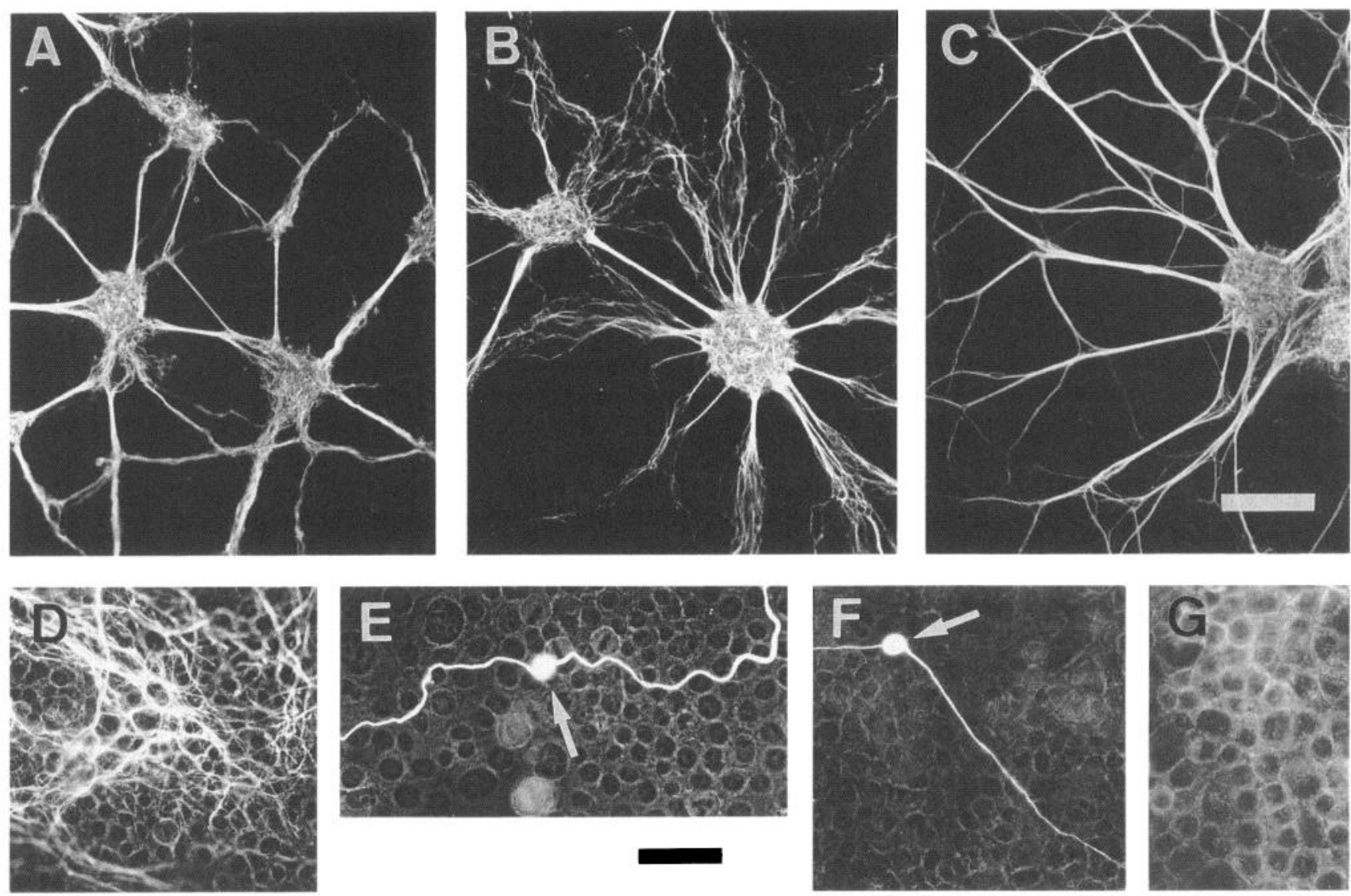

Figure 6. Axonal outgrowth from E6 (stage 28-29) chicken explants on N-cadherin-transfected and control neuroblastoma cells. Neurites are visualized by immunofluorescent staining with antibodies against neurofilaments. In $D-F$, the underlying monolayer is visualized by superimposing the phase-contrast images onto the neurofilament images. $A$, Outgrowth on the $\mathrm{N}$-cadherin-negative cell line $2 \mathrm{a}-01$ in the presence of $20 \mu \mathrm{g}$ of control IgG. $B$, Outgrowth on cell line $2 \mathrm{a}-\mathrm{Ncad} 1$, which expresses $\mathrm{N}$-cadherin, in the presence of $20 \mu \mathrm{g}$ of control IgG. $C$, Outgrowth on cell line $2 \mathrm{a}-\mathrm{Ncad} 1$ in the presence of $20 \mu \mathrm{g}$ of anti-N-cadherin IgG. Note that neurites are defasciculated in $B$ but not in $A$ and $C$. $D$, Higher magnification of the axonal outgrowth pattern on line 2a-Ncad $1 . E$ and $F$, Single sensory neurons growing on line 2a-Ncadl without $(E)$ and with $(F)$ the addition of $20 \mathrm{mg}$ of anti-N-cadherin IgG to the culture medium. The arrows point to the bodies of the bipolar sensory neurons. Note that the neurites in $E$ grow along the cell-cell boundaries whereas the neurites in $F$ grow straight. $G$, Line 2a-Ncadl stained for N-cadherin. High amounts of N-cadherin are expressed along the cell-cell boundaries. Scale bars: $C, 200 \mu \mathrm{m}$ for $A-C ; E, 50 \mu \mathrm{m}$ for $D-G$.

finding confirms studies in the chicken demonstrating that the medial part of the dorsal funiculus contains the longitudinal long-distance projections running up and down the spinal cord while the lateral part contains mostly short-distance projections (Davis et al., 1989). The N-cadherin-positive fibers likely correspond to the "external fascicle" of the dorsal root described by Ramon y Cajal and his contemporaries (Ramon y Cajal, 1911). This fascicle contains fine fibers that terminate, after a relatively short longitudinal run, in the dorsal horn of the spinal cord gray matter. The $\mathrm{N}$-cadherin-negative fibers may correspond to the "internal fascicle" (Ramon y Cajal, 1911), which contains large-diameter fibers giving rise to long-distance projections after bifurcating into ascending and descending branches. Thus, the $\mathrm{N}$-cadherin-positive and -negative regions of the dorsal funiculus constitute distinct domains in axon migration. A schematic summary of the immunostaining results and the neuroanatomical correlates is given in Figure $7 \mathrm{~A}$.

\section{$N$-cadherin is expressed in somatic sensory fibers of the cranial} nerves

In the brainstem, $\mathrm{N}$-cadherin is expressed in sensory fascicles of the trigeminal, glossopharyngeal, and vagus nerves (Fig. 3). The nerve cell bodies of these axons are located outside the brain in the respective ganglia. Inside the brain, these fibers together form the $\mathrm{N}$-cadherin-positive descending trigeminal tract (Figs. $3 B, 4 A$ ), which projects caudally and carries general somatic sensory information (Ramon y Cajal, 1911; Bok, 1915). We found that the $\mathrm{N}$-cadherin staining of this tract forms a continuous transition to the lateral part of the dorsal funiculus of the spinal cord. The fact that both tracts express $\mathrm{N}$-cadherin and their transition suggests that they share common developmental features.

The $\mathrm{N}$-cadherin-negative fibers of the major (sensory) portion of the trigeminal nerve (Fig. $4 C, D$ ) pass through the superficially located descending trigeminal tract. The minor portion of the trigeminal nerve, a separate fiber bundle located more ventrally, does not express $\mathrm{N}$-cadherin; it carries efferent (motor) neurites (Ramon y Cajal, 1911; Bok, 1915). Like the dorsal root of the spinal cord, the trigeminal nerve thus contains different fiber types of which the $\mathrm{N}$-cadherin-expressing fibers form a unique subset with a separate projection inside the CNS.

\section{Topographical coincidence of $\mathrm{N}$-cadherin expression and aberrant retinal projections}

The topographical distribution of $\mathrm{N}$-cadherin expression in the chicken CNS closely coincides with the paths taken by aberrant 

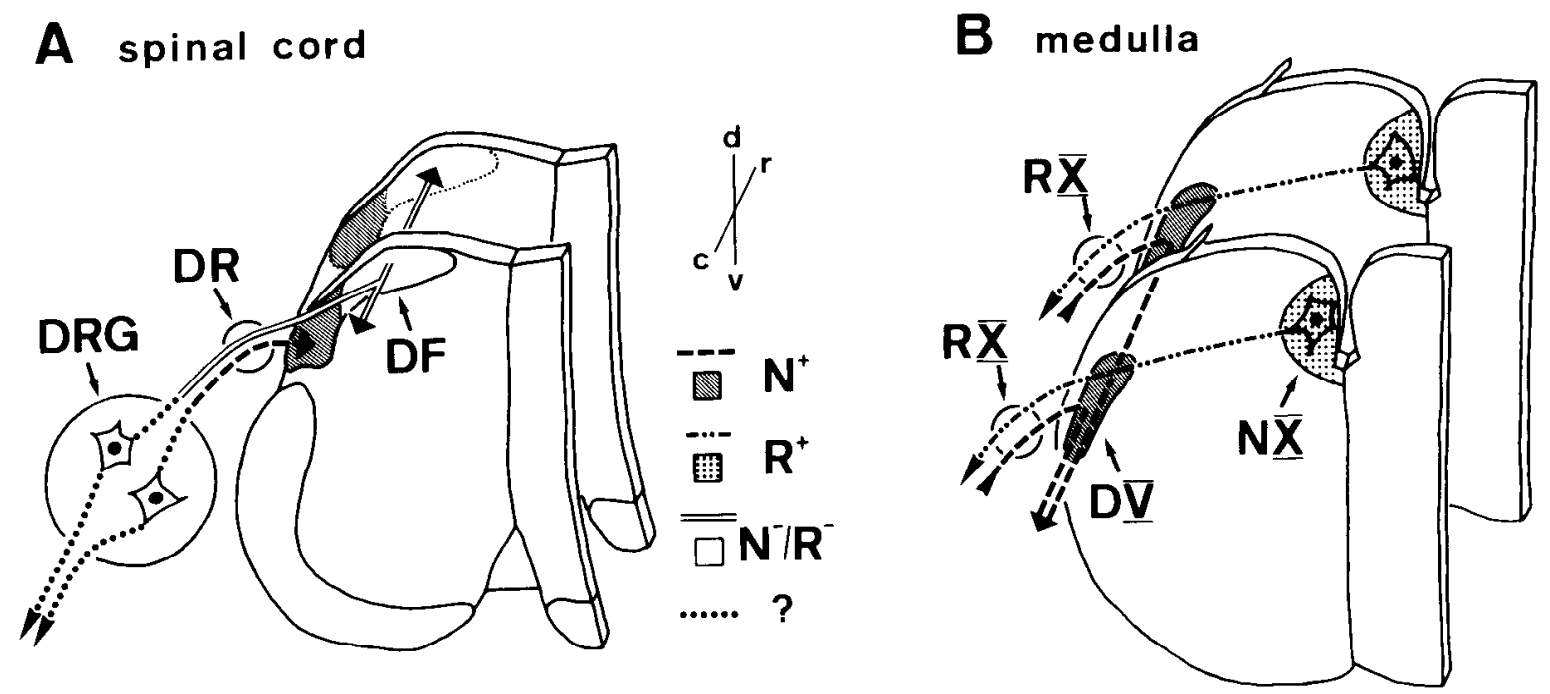

Figure 7. Schematic three-dimensional representation of the immunostaining results and their neuroanatomical correlates in the spinal cord $(A)$ and medulla $(B)$ of the chicken. Shadings indicate immunohistochemical staining results. Broken lines indicate the expression of N- or R-cadherin by hypothetical individual neurites, assuming that neurites in a fiber tract express the molecule detected in this tract immunohistochemically. $D R G$, dorsal root ganglion; $D R$, dorsal root; $D F$, dorsal funiculus; $d$, dorsal; $r$, rostral; $v$, ventral; $c$, caudal; $N+$, $N$-cadherin positive; $R+$, R-cadherin positive; $N-/ R-$, negative for both $\mathrm{N}$ - and R-cadherin; ?, immunostaining pattern unknown; $R X$, root of vagus nerve; $D V$, descending trigeminal tract; $N X$, motor nucleus of the vagus nerve.

projections from transplanted retinas induced in the frog (Constantine-Paton and Capranica, 1976; Constantine-Paton, 1978). In the brainstem, the aberrant projections follow the course of the descending trigeminal tract, and in the spinal cord, they typically grow in the dorsolateral, but not dorsomedial, white matter tract (Constantine-Paton, 1978). In the chicken, both tracts express $\mathrm{N}$-cadherin (Figs. 1, 3, 4A), as do retinal axons (Matsunaga et al., 1988a). If one assumes that frog and chicken are similar in their expression of $\mathrm{N}$-cadherin, these findings may indicate that $\mathrm{N}$-cadherin serves to guide vertebrate retinal axons along the aberrant pathways by a homophilic adhesion mechanism. This mechanism may work in conjunction with other molecules like A5 (Takagi et al., 1987, 1991), whose expression is similarly restricted to the developing retinotectal system and the descending trigeminal tract of the frog (Fujisawa et al., 1989).

\section{$N$-cadherin as a guidance cue for axon elongation}

$\mathrm{N}$-cadherin provides a potent substrate for axon outgrowth from retinal ganglion cells (Matsunaga et al., 1988; Drazba and Lemmon, 1990) and chick ciliary ganglion neurons (Tomaselli et al., 1988; Bixby and Zhang, 1990). Furthermore, Doherty et al. (1991) found a correlation between the length of rat cerebellar neurites and the level of $\mathrm{N}$-cadherin expression by the cells on which the neurites were growing. The restricted expression pattern of N-cadherin in the developing CNS (Figs. 1, 3) suggests that this molecule, like some members of the immunoglobulin superfamily (see introductory remarks), may provide a cue for the directed growth of axons along preformed, molecularly defined pathways.

Results from the present study support this hypothesis. The fact that, on $\mathrm{N}$-cadherin-transfected cells, the sensory ncurites defasciculate and grow along the cell-cell boundaries of the underlying monolayer (Fig. 6) implies that the axons more strongly adhere to cell-cell boundaries than to themselves, perhaps because $\mathrm{N}$-cadherin is present in higher amounts at the boundaries than on the free surface of the transfected cells (Fig. 6G) and the sensory axons themselves. This finding strongly suggests that sensory axons can use $\mathrm{N}$-cadherin as a specific cell adhesion molecule in vivo. The segregation of $\mathrm{N}$-cadherin-positive and -negative sensory fibers, found in the present study, thus may depend at least partly on $\mathrm{N}$-cadherin-mediated specific cell-cell adhesion. For example, N-cadherin-positive sensory axons might selectively migrate on preexisting axons or on glial cells that express the same molecule, resulting in the formation of $\mathrm{N}$-cadherin-positive and-negative fascicles in the sensory tract of the spinal cord. The same mechanism could operate for the segregation of $\mathrm{N}$-cadherin-positive and -negative sensory fibers of the trigeminal nerve at their entry into the brain (Fig. $4 B, C$ ).

\section{A simple, cadherin-based model for the segregation of motor and sensory fibers of the vagus nerve}

It has been shown that, whilc $\mathrm{N}$-cadhcrin and $\mathrm{R}$-cadhcrin can mediate cell-cell aggregation via their heterophilic binding, both prefer their own type (Inuzuka et al., 1991a). It is therefore conceivable that $\mathrm{N}$ - and R-cadherin cause the segregation of two fiber types each expressing only one of these cadherins. Figure $7 B$ illustrates this point for the sensory and motor fibers of the vagus nerve. $\mathrm{R}$-cadherin, but not $\mathrm{N}$-cadherin, is expressed by the cell bodies of the visceral motor neurons located inside the CNS close to the midline. The R-cadherin-positive neurites originating from these nuclei traverse the brainstem and reach the superficially located region of the descending trigeminal tract that expresses $\mathrm{N}$-cadherin (Figs. 3, 5). Here, the R-cadherinpositive motor axons largely ignore the descending sensory tract and grow straight through this tract to exit the brain via the vagus nerve rootlets (Fig. 5) (Ramon y Cajal, 1911 ; Bok, 1915). On the other hand, when the N-cadherin-positive sensory fibers of the vagus nerve enter the brain, they encounter the $\mathrm{N}$-cadherin-positive descending trigeminal tract, which causes them to turn their direction along this tract and to segregate from the $\mathrm{R}$-cadherin-positive motor fibers.

The weaker, heterophilic adhesion mechanism between $\mathrm{N}$ and R-cadherin (Inuzuka et al., 1991a) may mediate the relatively loose apposition of $\mathrm{N}$-cadherin-positive sensory fascicles 
and R-cadherin-positive motor fascicles in the rootlets of the vagus nerve (Fig. $5 B-E$ ). In this context, it may be of interest that the N-cadherin-positive sensory axons can also use R-cadherin-transfected cells as a substrate for neurite outgrowth (Redies and Takeichi, unpublished observations).

Although axon guidance mechanisms may not be so simple and may be regulated by complex factors, the ideas presented here should be considered as possibilities that should be tested by more analytical methods in future studies.

\section{References}

Auerbach R, Kubai L, Knighton D, Folkman J (1974) A simple procedure for the long-term cultivation of chicken embryos. Dev Biol 41:391-394.

Bixby JL, Zhang R (1990) Purified N-cadherin is a potent substrate for the rapid induction of neurite outgrowth. J Cell Biol 1 10:12531260.

Bok ST (1915) Die Entwicklung der Hirnnerven und ihre zentralen Bahnen. Folia Neurobiol 15:475-565.

Burns FR, von Kannen S, Guy L, Raper JA, Kamholz J, Chang S (1991) DM-GRASP, a novel immunoglobulin superfamily axonal surface protein that supports neurite extension. Neuron 7:209-220.

Constantine-Paton M (1978) Central projections of anuran optic nerves penetrating hindbrain or spinal cord regions of the neural tube. Brain Res 158:31-43

Constantine-Paton M, Capranica RR (1976) Axonal guidance of developing optic nerves in the frog. J Comp Neurol 170:17-32.

Davis BM, Frank E, Johnson FA, Scott SA (1989) Development of central projections of lumbosacral sensory neurons in the chick. $J$ Comp Neurol 279:556-566.

de la Rosa EJ, Kayyem JF, Roman JM, Stierhof YD, Dreyer WJ, Schwarz U (1990) Topologically restricted appearance in the developing chick retinotectal system of Bravo, a neural surface protein: experimental modulation of environmental cues. J Cell Biol 111:3087-3096.

Detrick RJ, Dickey D, Kitner CR (1990) The effect of N-cadherin misexpression on morphogenesis in Xenopus embryos. Neuron 4: 493-506.

Dodd J, Morton SB, Karagogeos D, Yamamoto M, Jessell TM (1988) Spatial regulation of axonal glycoprotein expression on subsets of embryonic spinal neurons. Neuron 1:105-116.

Doherty P, Rowett LH, Moore SE, Mann DA, Walsh SW (1991) Neurite outgrowth in response to transfected N-CAM and N-cadherin reveals fundamental differences in neuronal responsiveness to CAMs. Neuron 6:247-248.

Drazba J, Lemmon V (1990) The role of cell adhesion molecules in neurite outgrowth on Müller cells. Dev Biol 138:82-93.

Fujimori T, Miyatani S, Takeichi M (1990) Ectopic expression of $\mathrm{N}$-cadherin perturbs histogenesis in Xenopus embryos. Development 110:97-104

Fujisawa H, Ohtsuki T, Takagi S, Tsuji T (1989) An aberrant retinal pathway and visual centers in Xenopus tadpoles share a common cell surface molecule, A5 antigen. Dev Biol 135:231-240.

Grenningloh G, Rehm EJ, Goodman CS (1991) Genetic analysis of growth cone guidance in Drosophila: fasciclin II functions as a neuronal recognition molecule. Cell: 67:45-57.

Hamburger V, Hamilton H (1951) A series of normal stages in the development of the chick embryo. J Morphol 88:49-92.

Harlow E, Lane D (1988) Antibodies. A laboratory manual. Cold Spring Harbor, NY: Cold Spring Harbor Laboratories.

Hatta K, Takeichi M (1986) Expression of $\mathrm{N}$-cadherin adhesion molecules associated with early morphogenetic events in chick development. Nature 320:447-449.

Hatta K, Takagi S, Fujisawa H, Takeichi M (1987) Spatial and temporal expression pattern of $\mathrm{N}$-cadherin cell adhesion molecules correlated with morphogenetic processes of chicken embryos. Dev Biol 120:215-227.

Hatta K, Nose A, Nagafuchi A, Takeichi M (1988) Cloning and expression of cDNA encoding a neural calcium-dependent cell adhesion molecule: its identity in the cadherin gene family. J Cell Biol 106 873-881.

Honig MG, Hume RI (1986) Fluorescent carbocyanine dyes allow living neurons of identified origin to be studied in long-term cultures. J Cell Biol 103:171-187.

Inuzuka H, Miyatani S, Takeichi M (1991a) R-cadherin: a novel $\mathrm{Ca}^{2+}$ dependent cell-cell adhesion molecule expressed in the retina. Neuron 7:69-79.

Inuzuka H, Redies C, Takeichi M (1991b) Differential expression of $\mathrm{R}$ - and N-cadherin in neural and mesodermal tissues during early chicken development. Development 113:959-967.

Katoh K, Takahashi Y, Hayashi S, Kondoh H (1987) Improved mammalian vectors for high expression of G418 resistance. Cell Struct Funct 12:575-580.

Letourneau PC, Shattuck TA, Roche FK, Takeichi M, Lemmon V (1990) Nerve growth cone migration onto Schwann cells involves the calcium-dependent adhesion molecule, N-cadherin. Dev Biol 138:430442

Matsunaga M, Hatta K, Nagafuchi A, Takeichi M (1988a) Guidance of optic nerve fibres by $\mathrm{N}$-cadherin adhesion molecules. Nature 334: 62-64.

Matsunaga M, Hatta K, Takeichi M (1988b) Role of N-cadherin cell adhesion molecules in the histogenesis of neural retina. Neuron 1: 289-295.

Napolitano EW, Venstrom K, Wheeler EF, Reichardt LF (1991) Molecular cloning and characterization of B-cadherin, a novel chick cadherin. J Cell Biol 113:893-905.

Patel NH, Snow PM, Goodman CS (1987) Characterization and cloning of fasciclin III: a glycoprotein expressed on a subset of neurons and axon pathways in Drosophila. Cell 48:975-988.

Ramon y Cajal S (1911) Histologie du système nerveux de l'homme des vertébrés, Vol 1, pp 496-509. Paris: Maloine. Reprint. Madrid: Maloine, 1972

Ranscht B, Dours-Zimmermann MT (1991) T-cadherin, a novel cadherin cell adhesion molecule in the nervous system lacks the conserved cytoplasmic region. Neuron 7:391-402.

Rathjen FG, Schachner M (1984) Immunocytological and biochemical characterization of a new neuronal cell surface component (L1 antigen) which is involved in cell adhesion. EMBO J 3:1-10.

Rutishauser U, Jessell TM (1988) Cell adhesion molecules in vertebrate neural development. Physiol Rev 68:819-857.

Shiga T, Oppenheim RW (1991) Immunolocalization studies of putative guidance molecules used by axons and growth cones of intersegmental interneurons in the chick embryo spinal cord. J Comp Neurol 310:234-252.

Suzuki S, Sano K, Tanihara H (1991) Diversity of the cadherin family: evidence for eight new cadherins in nervous tissue. Cell Regul 2:261270.

Takagi S, Tsuji T, Amagai T, Takamatsu T, Fujisawa H (1987) Specific cell surface labels in the visual centers of Xenopus laevis tadpole identified using monoclonal antibodies. Dev Biol 122:90-100.

Takagi S, Hirata T, Agata K, Mochii M, Eguchi G, Fujisawa H (1991) The A5 antigen, a candidate for the neuronal recognition molecule, has homologies to complement components and coagulation factors. Neuron 7:295-307.

Takeichi M (1988) The cadherins: cell-cell adhesion molecules controlling animal morphogenesis. Development 102:639-655.

Takeichi M (1991) Cadherin cell adhesion receptors as a morphogenic regulator. Science 251:1451-1455.

Takeichi M, Inuzuka H, Shimamura K, Fujimori T, Nagafuchi A (1991) Cadherin subclasses: differential expression and their roles in neural morphogenesis. Cold Spring Harbor Symp Quant Biol 55:319-325.

Tanaka H, Matsui T, Agata A, Tomura M, Kubota I, McFarland KC, Kohr B, Lee A, Phillips HS, Shelton DL (1991) Molecular cloning and expression of a novel adhesion molecule, SC1. Neuron 7:535545.

Tomaselli KJ, Neugebauer KM, Bixby JL, Lilien J, Reichardt LF (1988) $\mathrm{N}$-cadherin and integrins: two receptor systems that mediate neuronal process outgrowth on astrocyte surfaces. Neuron 1:33-43. 